\title{
Using the profiled elements to increase electrocyclone effectiveness
}

\author{
Anton Trinkunas , Lidia Pomortseva, \\ Anatolii Titov, Zalina Rusinova \\ Ural Federal University, 19 Mira st., Ekaterinburg, 620002, Russia \\ *email:trinkunas.anton@yandex.ru
}

\begin{abstract}
The article is devoted to flue gas cleaning, using fly ash as an example. Electrocyclone can be employed as gas-cleaning equipment. The electrocyclone operation can be intensified by reducing re-entrainment. The laboratory model of the annular channel of the electrocyclone is used to show the possibility of reducing re-entrainment by using the shaped elements of various designs on the collecting electrodes. Aerosol industrial emissions can be reduced by using an electrocyclone with modified collecting electrodes.
\end{abstract}

Keywords: gas treatment; re-entrainment; shaped elements; electrocyclone

Received: 18.10.2020. Accepted: 07.12.2020. Published:30.12.2020.

(C) Anton Trinkunas, Lidia Pomortseva, Anatolii Titov, Zalina Rusinova, 2020

\section{Introduction}

Technological advancement im-

plies a continuous increase in the capacities of industrial companies. Industrial enterprises use solid materials for production purposes. Many of the thermal power stations supplying electricity to industrial companies also operate on solid fuels. Increasing volumes of emitted flue gases require high-performance gas-cleaning devices $[1,2]$.

Aerosols can be purified in various types of devices [3-5]. An electrocyclone is a combined dust collector that combines the centrifugal and electrostatic effects for cleaning aerosols. There has been unreasonably little focus on the electrocyclone research. A comprehensive study of this type of gas cleaning devices could lead to the introduction into the industry of a new type of highly efficient gas cleaning devices.

It is possible to intensify the operation of electrocyclones by optimising the various parameters and characteristics. The main of this is reduction of re-entrainment [6]. This work is devoted to the intensification of the work of the electrocyclone by reducing re-entrainment. The effectiveness of the profiled elements of different shapes is considered.

\section{Experimental}

For visual observation and quantification of the effectiveness of the profiled elements, a stand was assembled. A schematic diagram of the experimental stand

is shown in Fig. 1. The stand is designed as a prototype (in a first approximation) of the annular channel model. 
The stand consist of: 1 is the corona electrode, 2 is the external collecting electrode, 3 is the internal collecting electrode, 4 is the body, 5 is the collector, 6 is the inlet pipe, 7 is the exhaust pipe, 8 is the glass plate, 9 is the settling chamber. The walls (smooth aluminum plates) were connected to the positive pole of an external source of high voltage and grounded. The discharge electrodes are connected to the negative pole of the external highvoltage source.

4 most common structural elements were selected (Fig. 2): C-shaped element (a), double C-shaped element (b), rounded C-shaped elements (c), Z -shaped elements (d).

Re-entrainment is associated with ejection of the entrapped materials on the collecting electrodes. Fig. 3 shows a top view of the settling chamber with a besieged ash layer. It can be seen that on the deposited layer there are jagged areas.

The photo taken from the side shows that the largest number of holes are op- posite the fixed points of the corona discharge. The same can be said about other areas. In all cases, the observed jags are opposite the corona electrode, namely in front of the needles located thereon.

The shape of the holes is irregular, and they do not form a perfect cone or any spherical/elliptical surface. Such an irregular shape of the edge and surface of holes due to large particles hitting the material layer at an angle rather than strictly in a radial manner.

Numerous traces of particles in front of the fixed points of the corona discharge can be attributed to the high concentration of charged ions moving to the collecting electrode and the greater intensity of the electric field in that domain. A larger concentration of ions and larger electric field strength contribute to increasing the maximum possible charge as well as more intense particle charging. This increases the rate of the large particles migration to the collecting electrode by a few to a few dozen times.
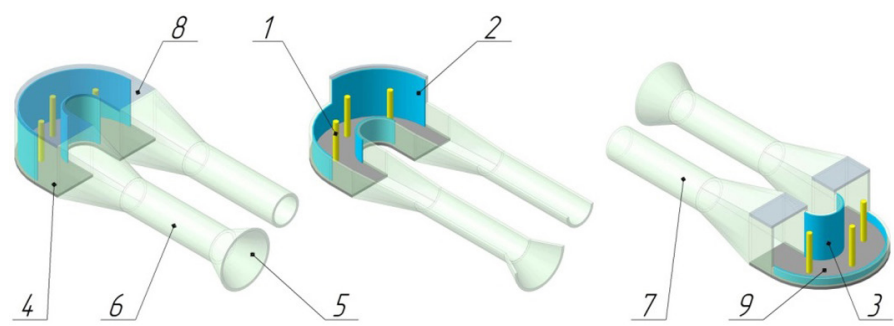

Fig. 1. A stand for the re-entrainment process study
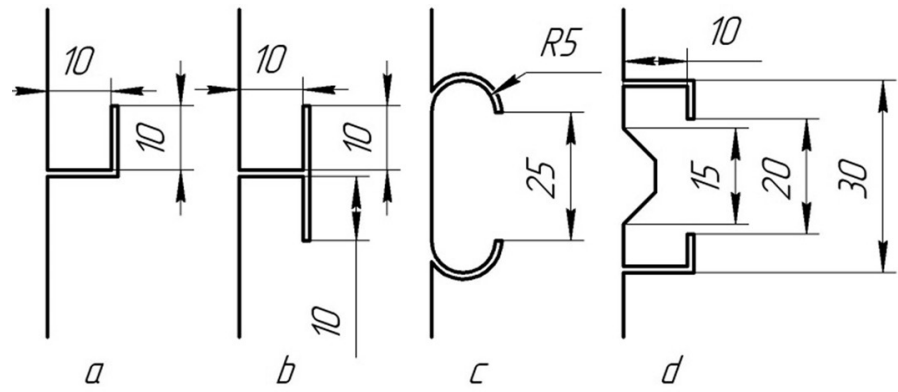

Fig. 2. Profiled elements 
The second part of the study was the visual and partly quantitative evaluation of the installation of profiled elements on the external collecting electrodes of the annular channel. Photographs of the profiled elements placed in the model of the active area channel with ashes settled on them are shown in Fig. 4. The ash was deposited on the surface of the collecting electrodes and the surface of the profiled elements. Moreover, the ash was on the outside and inside surfaces of the profiled elements.

Ash distribution on the external collecting electrodes is the same in all cases. Parts of low spots of the Z-shaped profiled elements are covered by a thicker layer of material, although there were areas not covered with ashes due to partial shielding of the corona discharge. Visually, the ash layer thickness on the electrodes seemed larger in all cases of the profiled elements, while the thickness of the layer on the smooth settling electrode without any elements seemed to be thinner. On the stand the dust collection efficiency was also quantitatively studied in the presence of the profiled elements in the active area channel. Evaluated was the amount of material accumulated on the collecting electrodes by passing a sample of $50 \mathrm{~g}$ of ash (Table 1).

It is shown that the most effective (13.8 g per 1 element) are double C-shaped elements; the lowest efficiency is demonstrated by C-shaped elements along the gas (13.0 g). Z-shaped ones catch only $11.3 \mathrm{~g}$ and the rounded $\mathrm{C}$-shaped ones catch $5.6 \mathrm{~g}$. This can be explained by the developed parasitic vortices in the radial cavity of the blades and between the blades.

Anyway, the profiled elements can reduce re-entrainment. This happens through the removal of large particles of the aerosol flow path due to delays and deposition and at expense of conservation on the surface of the particle layer in view of the wind shadow.

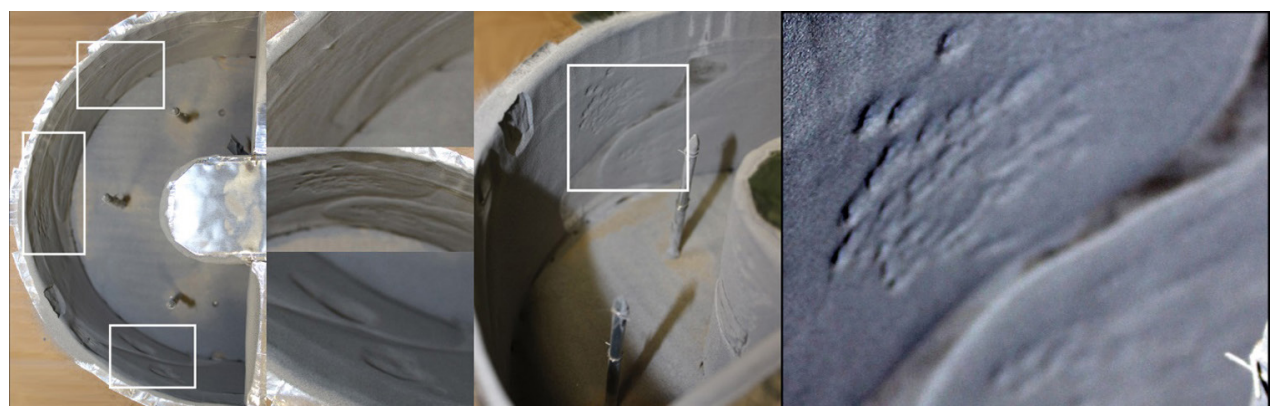

Fig. 3. Photograph of the deposited material layer. Channel without the profiled elements

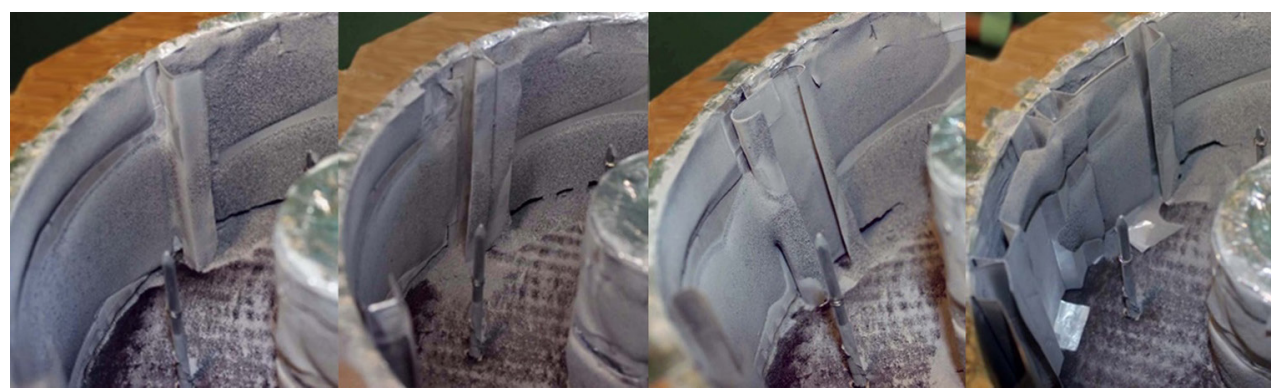

Fig. 4. C Photograph of the deposited material layer. Channel with the profiled elements 
Comparative effectiveness of the profiled elements

Table 1

\begin{tabular}{c|l|c|c|c}
\hline № & \multicolumn{1}{|c|}{ Element type } & $\begin{array}{c}\text { Collected ash } \\
\text { mass, g }\end{array}$ & $\begin{array}{c}\text { Quantity } \\
\text { of elements, piece }\end{array}$ & $\begin{array}{c}\text { Collected ash mass } \\
\text { corresponding on 1 element, g }\end{array}$ \\
\hline 1 & No elements & 16.2 & 2 & 8.1 \\
\hline 2 & C-shaped & 26.0 & 2 & 13.0 \\
\hline 4 & C-shaped double & 27.6 & 2 & 13.8 \\
\hline 5 & $\begin{array}{l}\text { C-shaped } \\
\text { rounded }\end{array}$ & 22.4 & 4 & 5.6 \\
\hline 6 & Z-shaped & 34.0 & 3 & 11.3 \\
\hline
\end{tabular}

\section{Conclusions}

Some research was conducted in a gas treatment process of the annular channel model of an electrocyclone. To assess the effectiveness of the profiled elements, an experimental stand was manufactured and exploratory tests were conducted. The design of the stand can be used for further experimentation.

\section{References}

1. Yan P, Zheng C, Zhu W, Xu X et al. An experimental study on the effects of temperature and pressure on negative corona discharge in high-temperature ESPs. Applied Energy. 2016;164:28-35.

doi:10.1016/j.apenergy.2015.11.040

2. Oh J, Choi S, Kim J. Numerical simulation of an internal flow field in a uniflow cyclone separator. Powder Technology. 2015;274:135-45.

doi:10.1016/j.powtec.2015.01.015

3. Chen CJ, Wang LFS. Cost-benefit analysis of electrocyclone and cyclone. Resources, Conservation and Recycling. 2001;31:285-92.

doi:10.1016/S0921-3449(00)00086-0

4. Zhuohan L, Shao C, An Y. et al. Energy-saving optimal control for a factual electrostatic precipitator with multiple electric-field stages based on GA. Journal of Process Control. 2013;23(8):1041-51.

doi:10.1016/j.jprocont.2013.06.007

5. Shrimpton JS, Crane RI. Small Electrocyclone Performance. Chemical Engineering \& Technology. 2001;24(9):951-5. doi:10.1002/1521-4125(200109)24:9<951::AID-CEAT951>3.0.CO;2-9

6. Titov A. The impact of re-entrainment on the electrocyclone effectiveness. Separation and Purification Technology. 2015;156(2):795-802.

doi:10.1016/j.seppur.2015.11.004 